\title{
FIRST ORDER PHASE TRANSITIONS INDUCED BY MAGNETIC FLUCTUATIONS AND DIMENSIONAL CROSSOVER IN TYPE I SUPERCONDUCTOR FILMS
}

\author{
J. C. Rahola \\ CPCM Laboratory, G. Nadjakov Institute of Solid State Physics \\ Bulgarian Academy of Sciences, BG-1784 Sofia, Bulgaria
}

(Received October 2, 2001)

\begin{abstract}
The crossover from a quasi-two dimensional to three dimensional thermodynamic behaviour near the point of the superconducting phase transition in type I superconductors is discussed with the help of a theoretical approach in which the fluctuations of the superconducting order parameter are neglected but the magnetic fluctuations are taken into consideration.
\end{abstract}

Key words: superconductivity, magnetic fluctuations, order parameter, phase transition.

PACS number(s): 64.60.Fr, 74.20.De, 74.30.Ek

I.

Under certain conditions three- and four-dimensional (3D and 4D) type I superconductors in zero external magnetic field undergo a "fluctuation-induced weaklyfirst order phase transition" from normal to superconducting (Meissner) state $[1,2]$. The interesting point is that this result contradicts to basic concepts about the kind of the superconducting phase transition. The normal-to-superconducting phase transition in type I superconductors is traditionally considered as one of the best examples of second order phase transitions described well by the mean field approximation [3]. However, it has been shown in Refs. [1,2] both by "mean-fieldlike" and renormalization group [3] arguments that the traditional notion for the second order of the superconducting phase transition is valid only within the standard mean field variant of the Ginzburg-Landau (GL) theory of superconductivity. The consideration of the magnetic fluctuations $\delta \mathbf{H}$ around the zero mean value $\mathbf{H}_{0}=(\mathbf{H}-\delta \mathbf{H})=0$ of the magnetic field (induction) $\mathbf{H}$ leads to the prediction [1] for a weakly-first order phase transition, i. e., a first order phase transition with a very small (experimentally unmeasurable) latent heat at the phase transition point at zero magnetic field $T_{C 0} \equiv T_{C}\left(H_{0}=0\right)$. Apparently this "weakly first order transition" is generated by the magnetic fluctuations $\delta \mathbf{H}$ in type I superconductors, where these fluctuations are stronger than the fluctuations of the superconducting order parameter $\psi(\mathbf{x})$, i. e., the London penetration depth $\lambda$ is much smaller than the coherence length $\xi$; the GL parameter $\kappa=(\lambda / \xi)$ is a small number $(\kappa \ll 1)$.

Using the condition $\kappa \ll 1$ one can apply "a meanfield-like" approximation [4]. Within this approximation the spatial $(\mathbf{x})$-dependence in the order parameter $\psi(\mathbf{x})$ is neglected. Moreover, the order parameter is substituted with its mean-field value: $\psi(\mathbf{x}) \approx \psi \equiv \psi_{\mathrm{MF}}$. This leads to a simplified version of the GL free energy and gives the opportunity for an exact integration of the vector potential $\mathbf{A}(\mathbf{x})$ of the magnetic field from the functional integral for the partition function — a method used in the scalar electrodynamics [4] and applied to the GL functional of superconductors $[1,2])$; here $\mathbf{A}(\mathbf{x})$ describes magnetic fluctuations, namely, $\mathbf{A}(\mathbf{x}) \equiv \delta \mathbf{A}(\mathbf{x})$, $\delta \mathbf{H}=(\nabla \times \delta \mathbf{A})$. Integrating over the field $\mathbf{A}(\mathbf{x})$ in the partition function and taking into account the Coulomb gauge $\operatorname{div} \mathbf{A}=0$, one obtains an effective free energy $F_{\text {eff }}(\psi)[1,2]$ which is a function of the uniform order parameter $\psi \equiv \psi_{\mathrm{MF}} \approx \psi(\mathbf{x})$. The effective free energy $F_{\text {eff }}(\psi)$ contains terms which include the effect of the magnetic fluctuations. The mathematical form of these terms depends mainly on the spatial dimensionality $D$. The analysis of the effective free energy $F_{\text {eff }}(\psi)$ leads to the prediction for the above mentioned weakly first order transition at spatial dimensionalities $D=3,4$; see Refs. [1, 2]. However, the concrete thermodynamical properties of $3 \mathrm{D}$ and 4D superconductors are quite different.

In Ref. [5] the prediction for a first order of the superconducting phase transition was extended to the $2 \mathrm{D}$ case - monoatomic superconducting layer. This could be true provided one has reasons to assume that the Meissner phase in the respective 2D (mono-atomic) superconducting layers is not destroyed by the strong $\psi$ fluctuations. Therefore the prediction in Ref. [5] should be taken with some caution.

In order to avoid the strong distructive effect of the $\psi$-fluctuations in monoatomic (exactly 2D) layers, a recent investigation [6] has been performed for quasi-2D (q2D) films of thickness $L_{0}$ which exceeds the lattice constant $a_{0}: L_{0} \gg a_{0}$. As a result of the mean-field-like approximation, the authors of paper [6] have obtained an interesting result that the latent heat of the fluctuationinduced first order phase transition in q2D superconducting films is large enough for a successful experimental observation in certain superconducting thin films, namely, that we are faced with a first order phase transition of a usual size rather than with a weak effect.

The effect of weakly first order phase transition in superconductors has been investigated also by renormalization group methods; see, e. g., the review article [7]. The same effect has been predicted for phase transitions 
in other systems such as early universe phase transitions [8, 9], and the nematic-smectic A phase transition in liquid crystals [10-12].

\section{II.}

In this note we outline a relatively simple scheme of treatment of the q2D-3D dimensional crossover within the framework of the mean-field-like approximation. We shall consider a superconducting slab with a finite thickness $L_{0}$ along one of the spatial directions (for example, the $z$-direction) and infinite sizes along the other $(D-1)$ directions of the $D$-dimensional space. At a certain stage of our investigation we shall use the practicaly important value $D=3$.

Varying the film thickness $L_{0}$ from relatively small values $a_{0} \ll L_{0} \ll \lambda$ to values exceeding the coherence length $\xi$ one may ensure the $\mathrm{q} 2 \mathrm{D}-3 \mathrm{D}$ crossover in real superconducting films. In order to avoid certain mathematical difficulties related with the finite-size scaling theory [13] we neglect the effect of the crystal structure by allowing variations of the wave vector $k$ outside the first Brillouihn zone up to infinity $(-\infty<\mathbf{k}<\infty)$ [14]. In a close vicinity of the phase transition point only the modes $A(\mathbf{k})$ with small wave vectors $(\mathbf{k} \sim 0)$ are important for the thermodynamic behaviour and this is the reason to assume that the above mentioned approximation will not affect our main results. For this reason we follow the method introduced in Ref. [14].

We derive the effective free energy for a general value of the dimensionality $D$, and then we focus our attention to the concrete problems of the q2D-3D crossover. Moreover, we present for the first time a graphical picture of the original results [1] for $3 \mathrm{D}$ superconductors and point out a minor error in Refs. $[1,2]$.

III.

For a spatially uniform order parameter $\psi$, the Ginzburg-Landau (GL) free energy takes the form [6]

$$
\begin{aligned}
& F_{G L}=V\left(a \varphi^{2}+\frac{b}{2} \varphi^{4}\right) \\
& +\frac{1}{16 \pi} \sum_{i, j=1}^{D} \int d^{D} x\left[2 \rho(\varphi) \delta_{i j} A_{j}^{2}+\left(\partial_{i} A_{j}-\partial_{j} A_{i}\right)^{2}\right]
\end{aligned}
$$

where $(i, j)=1, \ldots, D, V=\left(L_{1} \ldots L_{D}\right)$ is the volume of the $D$-dimensional superconductor, $\alpha=\alpha_{C 0}\left(T-T_{C 0}\right)$, $\rho=\rho_{0} \varphi^{2}$ with $\rho_{0}=8 \pi e^{2} / m_{e} c^{2}$, and $\varphi=|\psi|$. The critical temperature $T_{C 0}$ as well as the GL parameters $\alpha_{C 0}$ and $b$ are known from BCS theory [15]. Finally, $A_{i}$ denotes the components of the vector potential $\mathbf{A}$.

We consider well established type I superconductors with GL parameter $\kappa \sim 10^{-1} \div 10^{-2}$. On the other hand,
$t=\left(T-T_{C 0}\right) / T_{C 0}$ must be also much less than unity, $t \sim 10^{-2} \div 10^{-4}$, in order to ensure a validity of the GL theory of type I superconductors [15]. A good example of a superconductor obeying the above requirements is Al.

Let us introduce the usual notations of the London penetration depth $\lambda(T)=\lambda_{0} /|t|^{1 / 2}$ and the coherence length $\xi(T)=\xi_{0} /|t|^{1 / 2}$, where $\lambda_{0}=$ $\left(m_{e} c^{2} b / 8 \pi e^{2} \alpha_{0} T_{C 0}\right)^{1}{ }_{2}$ and $\xi_{0}=\left(\hbar^{2} / 4 m_{e} \alpha_{0} T_{C 0}\right)^{1 / 2}$ are the zero-temperature penetration depth and the zerotemperature coherence length, respectively. In order to ensure validity of our consideration we assume the following relation between the characteristic lengths: characteristic lengths $\lambda(T) \gg \xi(T) \gg \xi_{0}[6]$. The irrelevance of the large values of the wave number $k=|\mathbf{k}|$ allows for the choice $\Lambda \sim 1 / \xi_{0}$ for the upper cutoff $\Lambda: k<\Lambda$. We use this cutoff for the wave vector component $\mathbf{k}_{\|}$along the large ("infinite") slab dimensions whereas we expand the cutoff to infinity for the wave vector component $\mathbf{k}_{\perp}$ along the small size $L_{0} ; \mathbf{k}=\left(\mathbf{k}_{\|}+\mathbf{k}_{\perp}\right)$.

Following Refs. [4-6] we calculate the effective free energy $f_{\text {eff }}(\psi)=F_{\text {eff }}(\psi) / V$ with the help of the expression $F_{\text {eff }}=-k_{B} T \ln Z$, where $Z$ is a partition function given by the functional integral

$$
Z(\varphi)=\int D \mathbf{A}(\mathbf{x}) \delta[\operatorname{div} \mathbf{A}(\mathbf{x})] \exp \left\{-F_{\mathrm{GL}}[\psi, \mathbf{A}(\mathbf{x})]\right\}
$$

The integral for $Z$ can be exactly solved by an expansion in power series of $\rho(\varphi)$. The result is an infinite logarithmic series that is summed up completely to give the result

$$
\begin{aligned}
f_{\mathrm{eff}}(\varphi) & =a \varphi^{2}+b \varphi^{4} \\
& +\frac{(D-1)}{2 V} k_{B} T \sum_{k} \ln \left[1+\rho(\varphi) / k^{2}\right] .
\end{aligned}
$$

For thin films the continuum limit $\left(\sum \longrightarrow \int\right)$ in Eq. (3) can be taken only for the longitudinal wave vector components $\left(\mathbf{k}_{\|}\right)$. The summation over the transverse wave vector component $\left(-\infty<\mathbf{k}_{\perp}<\infty\right)$ should be performed in the form given by Eq. (3).

For $D=3$, we obtain the effective free energy density in the form

$$
\begin{aligned}
f_{\mathrm{eff}}(\varphi) & =a \varphi^{2}+\frac{b}{2} \varphi^{4}+\frac{k_{B} T}{2 \pi L_{0}} \\
& \times \int_{0}^{\Lambda} k_{\|} \ln \left[\sinh \left(\frac{L_{0}}{2} \sqrt{\rho(\varphi)+k_{\|}^{2}}\right)\right] d k_{\|} .
\end{aligned}
$$

The term associated with fluctuations depends on the thickness $L_{0}$ which means that this parameter will play some role in the thermodynamic properties. As we shall see the film thickness $L_{0}$ is the parameter that determines essential phase transition properties. 
The integration in the free energy (4) can not be performed analytically. Because of this reason and for testing the validity of our model two limiting cases are considered:

(i) $L_{0} \ll \lambda_{0}$ (q2D case, very thin film), and

(ii) $L_{0} \gg \lambda_{0}$ (3D case, thick film).

For the q2D film we obtain

$$
f_{\mathrm{eff}}(\varphi)=s_{0} \varphi^{2}+\frac{w}{2} \varphi^{4}-p_{0} \varphi^{2} \ln \left[\frac{\rho_{0} \varphi^{2}}{\Lambda^{2}}\right]
$$

where

$$
\begin{aligned}
& s_{0}=a+k_{B} T \rho_{0} / 4 \pi L_{0}, \\
& w=b+k_{B} T \rho_{0}^{2} / 4 \pi L_{0} \Lambda^{2},
\end{aligned}
$$

and

$$
p_{0}=k_{B} T \rho_{0} / 4 \pi L_{0} .
$$

Thus we have received a GL energy (5)-(8) with a logarithmic term which is typical for q2D systems [6]. This energy and its parameters are exactly the ones associated with the q2D system considered in Ref. [6]. Within this limiting case of extremely small thickness the $k$-sum in Eq. (3) is taken only for wave vector components parallel to the film surface, whereas the axial one, the wave numbers $k_{\perp} \neq 0$, are ignored because they give a small contribution. Of course, the same result (5)-(8) is obtained also by the approximation $\ln (\sinh z) \approx z$ in Eq. (4), which corresponds to $z \ll 1$, i. e., to the case (i): $L_{0} \ll \lambda_{0}$.

Using the parameters

$$
s=\left[s_{0}-p \ln \left(\rho_{0} / \Lambda^{2}\right)\right] / w
$$

and $p=p_{0} / w$, the free energy $f_{\text {eff }}(\varphi) / w \equiv f_{\text {eff }}$ takes the form:

$$
f_{\mathrm{eff}}=s \varphi^{2}+\frac{1}{2} \varphi^{4}-p \varphi^{2} \ln \left(\varphi^{2}\right)
$$

The shape of the free energy (10) is shown in Fig. 1 for several values of the parameters $s$ and $p$. Fig. 1 shows a well established first order phase transition for a certain range of temperatures. When overheating, the superconducting phase $(\varphi>0)$ becomes metastable in a narrow temperature interval above the equilibrium phase transition point. If the overheating continues the superconductor state disappears. The phases and their stability properties can be found [6] as solutions of the equation of state: $\left(\partial f_{\text {eff }} / \partial \varphi=0\right)$. The normal phase $\left(\varphi_{0}=0\right)$ appears as a minimum of energy at any temperature. The equilibrium order parameter $\varphi_{0}>0$ of the superconduct- ing phase is obtained from the equation

$$
(s-p)+\varphi_{0}^{2}-p \ln \varphi_{0}^{2}=0
$$

The solution of the Eq. (11) is shown in Fig. 2 for values of the parameters $s$ and $p$ corresponding to the size of the first order transition. The order parameter values belonging to stable superconduting states are shown together with their extension to metastable states. The jump of the order parameter to zero at the phase transition point is well established. These results confirm the predictions made in Ref. [6].

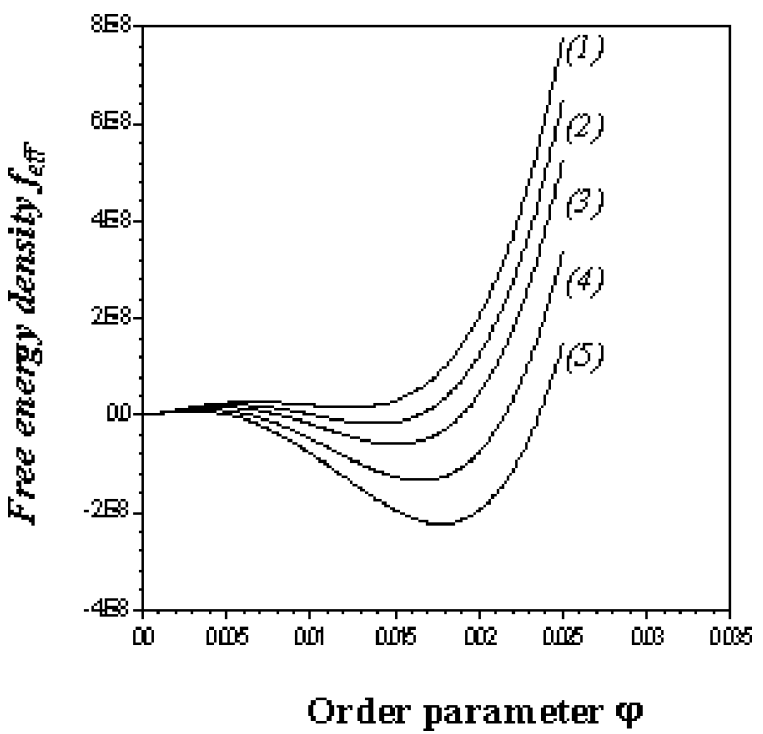

Fig. 1. Thin films free energy for $p=8.7 \cdot 10^{-5}$. Curve (1) corresponds to $s=-8.3 \cdot 10^{-4}$, curve $(2)-$ to $s=-8.5 \cdot 10^{-4}$, (3) $-s=-8.7 \cdot 10^{-4}$, (4) $-s=-9 \cdot 10^{-4}$, (5)$s=-9.3 \cdot 10^{-4}$.

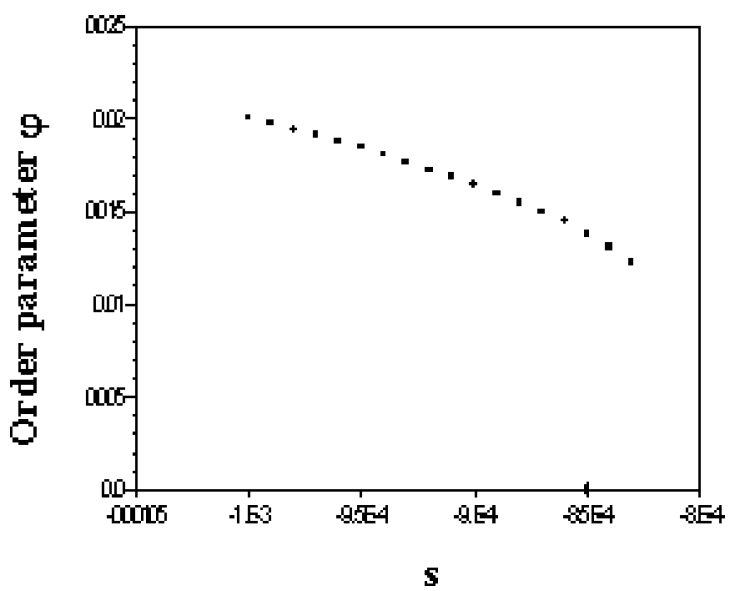

Fig. 2. Thin films order parameter profile for $p=8.7 \cdot 10^{-5}$. The crosses indicate stable superconducting states while the squares indicate metastable states above the equilibrium transition point. 
The second limiting case, that is the thick (3D) film $\left(L_{0} \gg \lambda\right)$, is considered in a similar way. Taking the continuum limit in Eq. (3) we obtain

$$
f_{\mathrm{eff}}(\varphi)=r_{0} \varphi^{2}-c_{0} \varphi^{3}+\frac{u}{2} \varphi^{4}
$$

with parameters

$$
\begin{aligned}
& r_{0}=a+k_{B} T \Lambda \rho_{0} / 8 \pi, \\
& u=b+k_{B} T \rho_{0}^{2} / 8 \pi \Lambda,
\end{aligned}
$$

and

$$
c_{0}=\left(k_{B} T / 12 \pi\right) \rho_{0}^{3 / 2}
$$

Eqs. (12)-(15) correspond to the result in Ref. [1] with the only difference that in our case we have taken into account the $\rho^{2}$-contribution to the parameter $u$. In Ref. [1] this contribution has been neglected. In Ref. [2] this fact was explained with the supposition that the respective term is very small. While the term neglected in Refs. [1,2] is irrelevant for the consideratiions made in the respective papers, the same term may be relevant for other calculations [16]. The results (12)-(15) can be obtained also from Eq. (4) by the approximation of the function $\ln (\sinh z)$ for $z \gg 1$, which corresponds to the limiting case (ii).

Using the parameters $r=r_{0} / u$ and $c=c_{0} / u$ the free energy $f_{\text {eff }}=f_{\text {eff }}(\varphi) / u$ becomes

$$
f_{\mathrm{eff}}=r \varphi^{2}-c \varphi^{3}+\frac{1}{2} \varphi^{4}
$$

This free energy is drawn in Fig. 3. Fig. 3 shows the typical local minimum corresponding to metastable superconducting states and, hence, describes a first order transition. Fig. 4 presents the profile of the equilibrium order parameter obtained by the equation

$$
r+\varphi_{0}^{2}-\frac{3}{2} c \varphi_{0}=0
$$

for the equilibrium order parameter of the superconducting phase $\varphi_{0}>0$. There is only a quantitative difference between Figs. 1 and 4 as well as between Figs. 2 and 4 .

The crossover between the two limiting cases, (i) and (ii), can be described with the help of Eq. (4). We have already demonstrated that Eq. (4) describes the limiting cases (i) and (ii).

The numerical analysis of the free energy (4) for var- ious values of the thickness $L_{0}$ is supposed to give the interpolation between the $\mathrm{q} 2 \mathrm{D}$ and $3 \mathrm{D}$ thermodynamical behaviour. It has been recently noted [16] that although Eq. (4) can hardly be treated analytically, the integral over $\mathbf{k}_{\|}$in the respective general equation of state, $[\partial f(\varphi) / \partial \varphi]=0$, can be solved exactly for $D=3$ and, therefore, some analytical predictions about the thermodynamic behaviour of the system can be made. Following this idea we obtain the equation for the superconducting order parameter $\left(\varphi_{0}>0\right)$ :

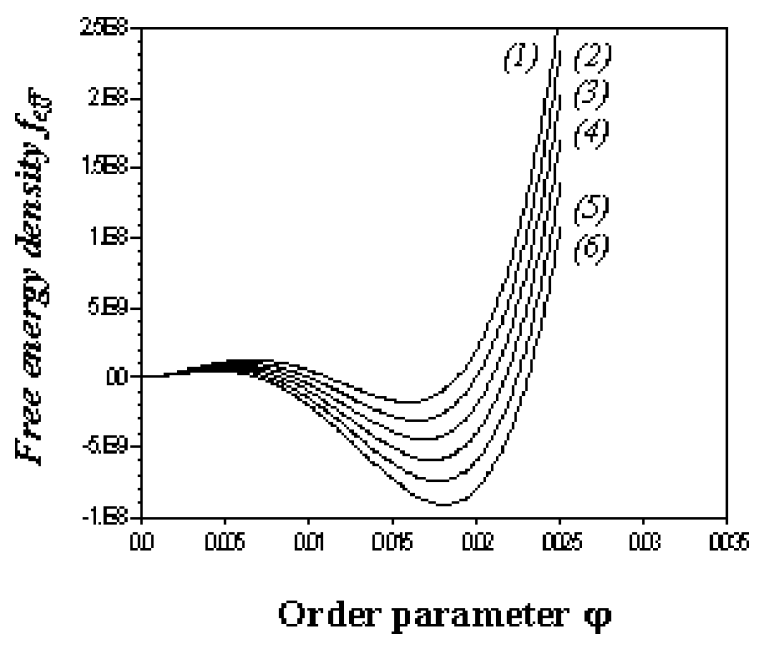

Fig. 3. Thick films free energy for $c=1.5 \cdot 10^{-2}$. Curve (1) corresponds to $r=1.05 \cdot 10-4$, (2) stands for $r=10^{-4}$, (3) $-r=9.5 \cdot 10^{-5},(4)-r=9 \cdot 10^{-5},(5)-r=8.5 \cdot 10^{-5}$, (6) $-r=8 \cdot 10^{-5}$.

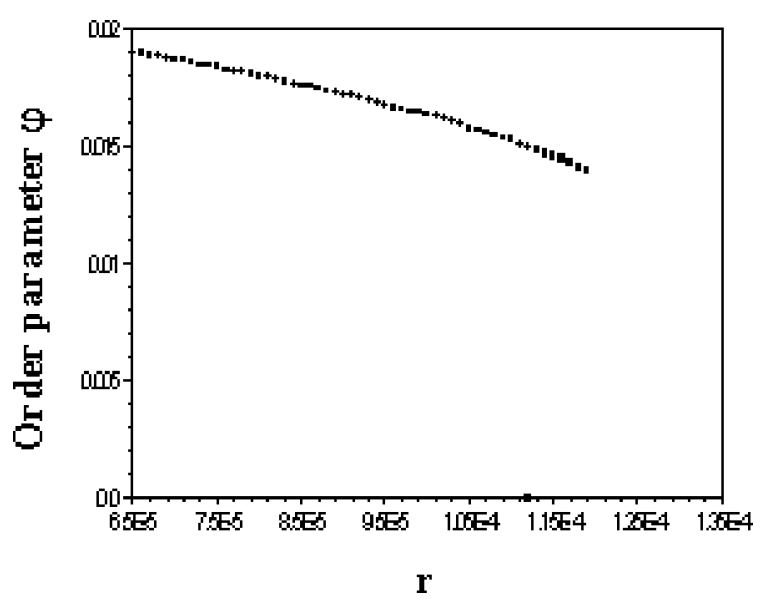

Fig. 4. Thick films order parameter profile for $c=1.5 \cdot 10^{-2}$. The crosses indicate the stable superconducting states and the squares stand for metastable states. 


$$
a+b \varphi_{0}^{2}+\frac{k_{B} T}{4 \pi L_{0}} \rho_{0} \ln \left[\frac{\sinh \left(\frac{L_{0} \Lambda}{2} \sqrt{1+\rho_{0} \varphi_{0}^{2} / \Lambda^{2}}\right)}{\sinh \left(L_{0} \varphi_{0} \sqrt{\rho} / 2\right)}\right]=0
$$

Using the condition for a stability of the phases, $\left[\partial^{2} f(\varphi) / \partial \varphi^{2}\right]>0$, and applying this condition to the equilibrium superconducting order parameter $\left(\varphi_{0}\right)$ we have

$$
4 b \varphi_{0}^{2}+\frac{k_{B} T \rho_{0}^{2} \varphi_{0}^{2}}{4 \pi}\left[\frac{\operatorname{cth}\left(\frac{L_{0}}{2} \sqrt{\Lambda^{2}+\rho_{0} \varphi_{0}^{2}}\right)}{\sqrt{\lambda^{2}+\rho_{0} \varphi_{0}^{2}}}-\frac{\operatorname{cth}\left(L_{0} \sqrt{\rho} \varphi_{0} / 2\right)}{\sqrt{\rho_{0}} \varphi_{0}}\right]>0 .
$$

The results (18) and (19) can be further analyzed numerically to demonstrate precisely the dependence of the thermodynamic behaviour on the thickness $L_{0}$. One may expect that the first order transition will take place for any thickness $L_{0}$ within the scope of validity of our consideration [6]. Besides, the numerical treatment of the results (4), (18), and (19) can be used to describe the gradual enhancement of the first order transition effect with the decrease of the length $L_{0}$ from values $L_{0} \gg \lambda$ corresponding to $3 \mathrm{D}$ films to values $L_{0} \ll \lambda$ corresponding to q2D films.

\section{IV.}

Note that our approach allows for a treatment of other relevant quantities such as the specific heat capacity, the latent heat, and the temperature size of this fluctuation induced first order phase transition $[1,6]$. Certainly, the analysis of these quantities will reveal the enhancement of the effects corresponding to a first order transition when the thickness $L_{0}$ of the superconducting films is lowered.

Finally, an experimental work could be performed to measure the size of the first order transition in thin superconducting films of various thicknesses and to compare the results with our theoretical predictions. As noted in Ref. [6] the size of the first order transition in thin superconducting films of thickness $L_{0} \sim 10^{-1} \mu \mathrm{m}$ is large enough to be observed experimentally. Therefore, our consideration may be used in interpretations of experimental results intended to observe the effect of the thickness variation on the properties of the fluctuationinduced first order phase transition in thin films.

\section{ACKNOWLEDGEMENTS}

The author thanks the hospitality of the Bulgarian Academy of Sciences during his six months stay for a specialization course. The author is grateful to Dr. Diana V. Shopova and Prof. Dimo I. Uzunov for numerous and valuable scientific discussions on problems of condensed matter theory and phase transitions.
[1] B. I. Halperin, T. C. Lubensky, S. K. Ma, Phys. Rev. Lett. 32, 292 (1974).

[2] J.-H. Chen, T. C. Lubensky, D. R. Nelson, Phys. Rev. B 17, 4274 (1978).

[3] D. I. Uzunov, Introduction to the Theory of Critical Phenomena (World Scientific Publishing, World Scientific, 1993).

[4] S. Coleman, E. Weinberg, Phys. Rev. D 7, 1888 (1973).

[5] S. W. Lovesey, Z. Phys. B 40117 (1980).

[6] R. Folk, D. V. Shopova, D. I. Uzunov, Phys. Lett. A 281 , 197 (2001).

[7] R. Folk, Yu. Holovatch, in Correaltions, Coherence, and Order, edited by D. V. Shopova, D. I. Uzunov (Kluwer Academic/Plenum Publ, London, 1999), p. 83.

[8] A. D. Linde, Rep. Prog. Phys. 42, 389 (1979).

[9] A. Vilenkin, E. P. S. Shellard, Cosmic Strings and Other
Topological Defects, Ch. 2 (Cambridge University Press, Cambridge, 1994).

[10] T. C. Lubensky, J.-H. Chen, Phys. Rev. B 17, 366 (1978).

[11] J. Toner, Phys Rev. B 26, 462 (1982).

[12] M. A. Anisimov, P. E. Cladis, E. E. Gorodetskii, D. A. Huse, V. E. Podneks, V. G. Taratuta, W. van Saarloos, V. P. Voronov, Phys. Rev. A 41, 6749 (1990).

[13] M. Barber, M. E. Fisher, Ann. Phys. 77, 1 (1973).

[14] L. Craco, L. De Cesare, I. Rabuffo, I. P. Takov, D. I. Uzunov, Pysica A 270, 486 (1999).

[15] E. M. Lifshitz, L. P. Pitaevskii, Statistical Physics (Landau and Lifshitz Course of Theoretical Physics, vol 9) (Nauka, Moscow, 1978) [Translated into English (Pergamon Press, Oxford, 1980)].

[16] D. I. Uzunov, privite communication (June 2001). 
FIRST ORDER PHASE TRANSITIONS INDUCED BY MAGNETIC FLUCTUATIONS ...

\title{
ФАЗОВІ ПЕРЕХОДИ ПЕРШОГО РОДУ, СПРИЧИНЕНІ МАГНЕТНИМИ ФЛУКТУАЩІЯМИ І КРОСОВЕР ЗА ВИМІРНІСТЮ В НАДПРОВІДНИХ ПЛІВКАХ
}

\author{
Й. К. Рагола \\ Лабораторія СРСМ, Інститут фізики твердого тіла ім. Г. Надюакова \\ Болаарсъка академіл наук, Софійсъкий університет, BG-1784, Софіл, Болааріл
}

$\mathrm{y}$ межах теоретичного підходу, у якому нехтуються флюктуації напівпровідникового параметра порядку, але приймаються до уваги магнетні флуктуації, обговорено кросовер із квазідвовимірної до тривимірної термодинамічної поведінки поблизу точки фазового переходу в надпровідний стан у напівпровідниках I ро,ту. 